\title{
The Histopathological Evaluation of Gallic Acid on Rat Purkinje Cells after Trimethyltin Intoxication
}

\author{
Lida Doroodi ${ }^{1,2}$, Mohammad Amin Edalatmanesh ${ }^{2 *}$ \\ 'Department of Physiology, College of Sciences, Fars Science and Research Branch, Islamic Azad University, Fars, Iran \\ ${ }^{2}$ Department of Physiology, College of Sciences, Shiraz Branch, Islamic Azad University, Shiraz, Iran
}

\section{A BSTRACT}

Introduction: Multiple studies have been shown neuroprotective effect of Gallic acid (GA) as a potent anti-oxidant substance. Furthermore, Trimethyltin (TMT) is a methylated organotin compound which induces neuronal degeneration in the brain of human and rodents. This study examined the effects of GA on wet weight of the cerebellum, cerebellum to total brain weight ratio, and purkinje cells number in lobule IV, V, and VI in the brain of TMT-intoxicated rats. Materials and Methods: Fifty adult male sprague dawley rats were divided into five groups; control, TMT+Saline group received saline after TMT $(8 \mathrm{mg} / \mathrm{Kg} / \mathrm{BW})$ intoxication as well as TMT+GA50, TMT+GA100 and TMT+GA200 groups. Animals received GA 50, 100 and $200 \mathrm{mg} / \mathrm{Kg}$ body weight seven days before and seven days after administration of TMT. Finally, through transcardialy perfusion, the rats were sacrificed and the weight of total brain and cerebellum was measured and then histopathological analyses were conducted. Results: The wet weight of cerebellum were significantly decreased in TMT+Saline group compared to controls and increased in GA-treated rats compared to TMT+Saline group. However, the average cerebellar to total brain weight ratio was not differed in different groups. The number of Purkinje cells in the specific folia was significantly increased in the GA-treated rats in comparison to that of the TMT+Saline group. Conclusion: Our data indicate that GA reduced TMT toxicity and associated cerebellar damages possibly via its antioxidant and neuroprotective properties.
Key words:
1. Cerebellum
2. Organotin Compounds
3. Polyphenols

*Corresponding Author: Mohammad Amin Edalatmanesh

E-mail: amin.edalatmanesh@gmail.com 


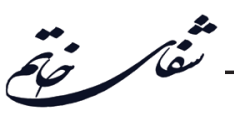

بررسى هيستو ياتولوزيك كاليك اسيد بر سلولهاى يوركينز موش صحرايى متعاقب مسموميت با ترى

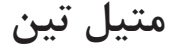

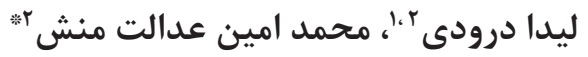 \\ 'كروه فيزيولوزى، دانشكده علوم، واحد علوم و تحقيقات فارس، دانشَاه آزاد اسلامى، فارس، ايران

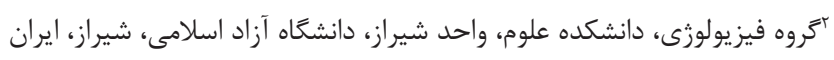

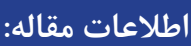

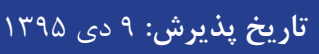

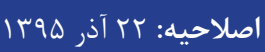

تاريخ دريافت: ه آذر هوسا

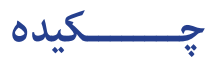

مقدمه: مطالعات متعدد نقش حمايت كننده عصبى كاليك اسيد را بلهنوان يك آنتى اكسيدان قوى نشان

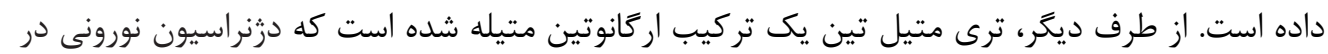

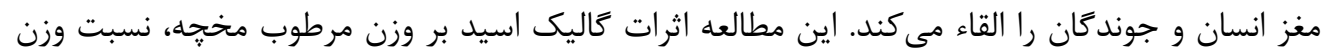

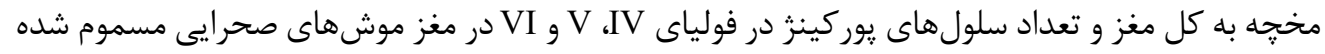

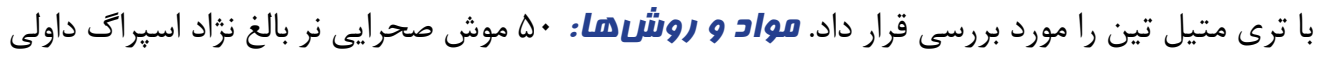

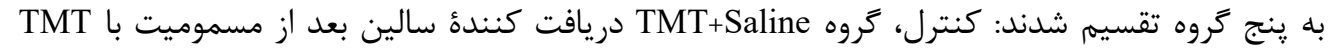

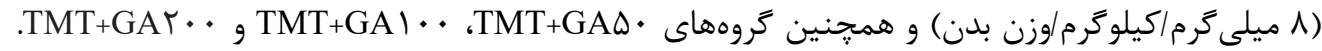

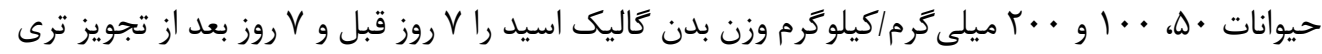

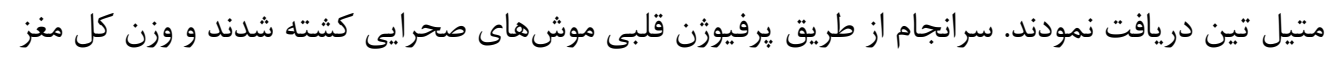

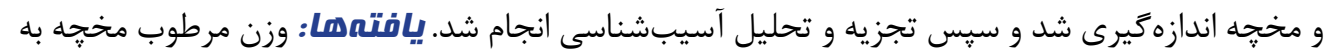

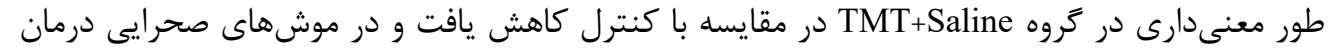

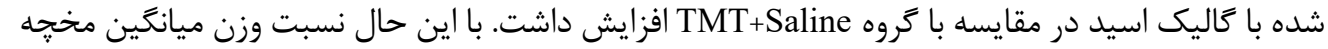

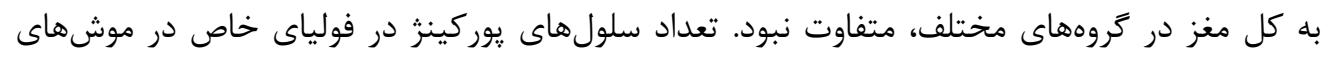

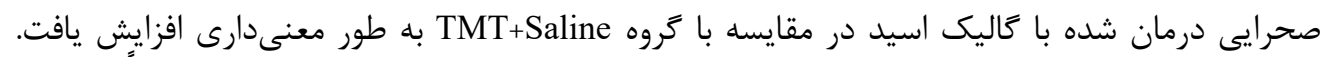

كليد وازهها:

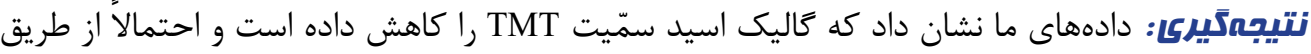

ו

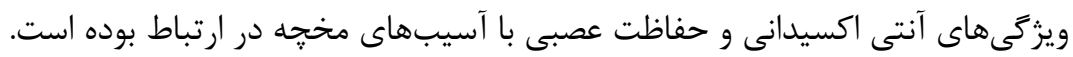

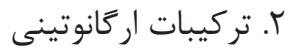

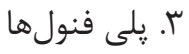

* نويسنده مسئول: محمد امين عدالت منش آدرس الكترونيكى: amin.edalatmanesh@gmail.com 


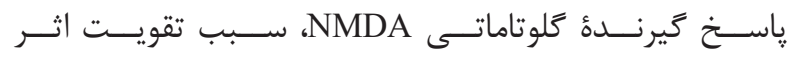

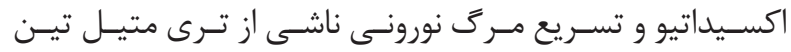

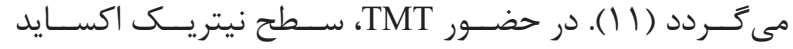

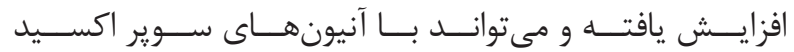

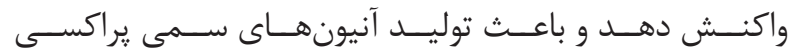

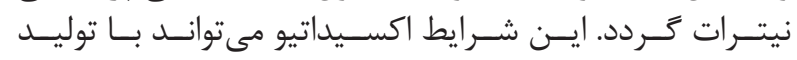

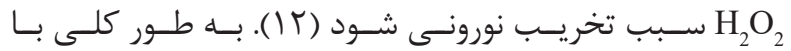

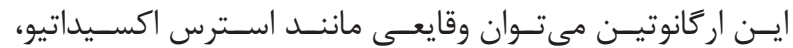

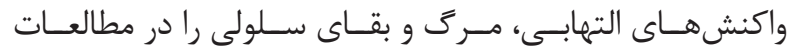

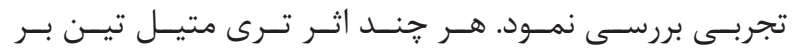

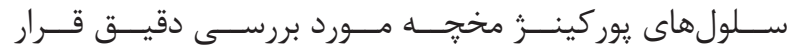

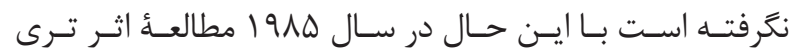

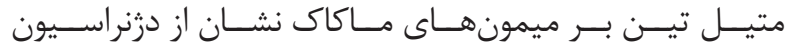

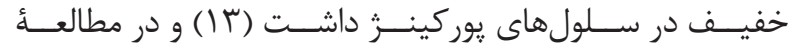

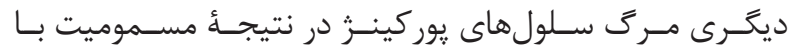

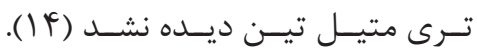

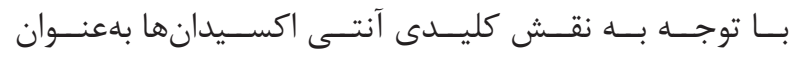

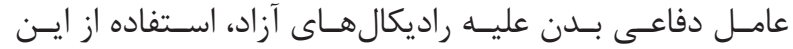

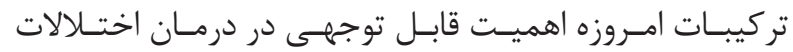

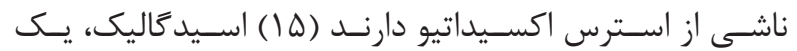

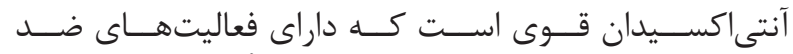

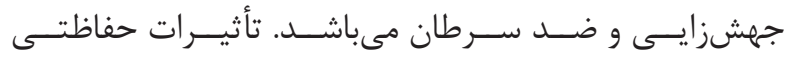

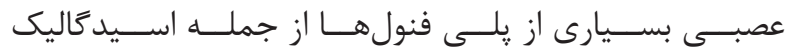

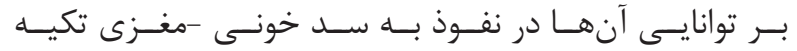

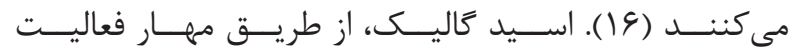

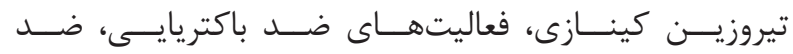

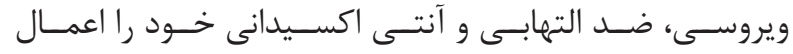

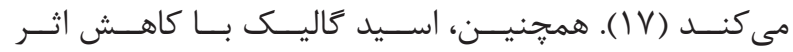

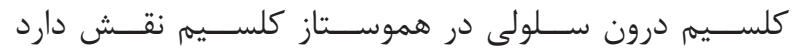

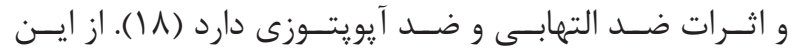

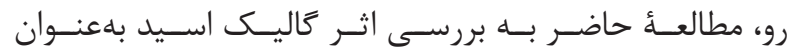

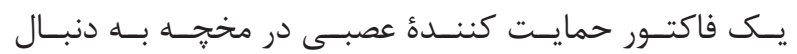
مســموميت تـــى متيـلـ تينــى مى فيــردازد.

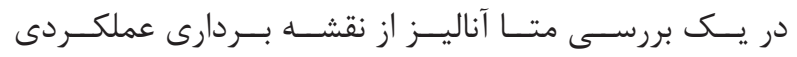

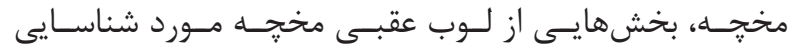

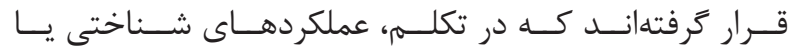

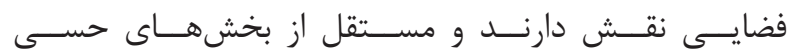

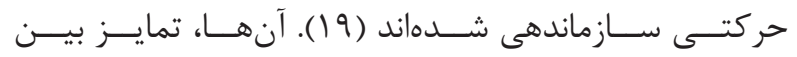

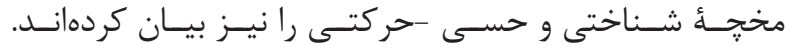

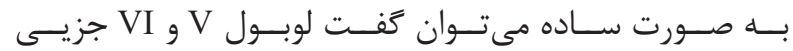

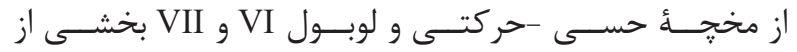

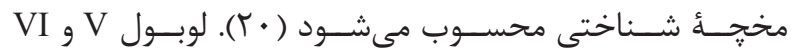

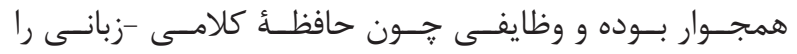

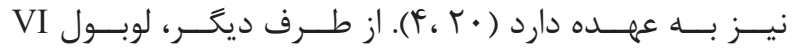

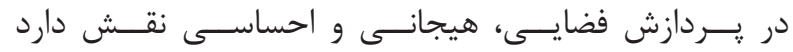

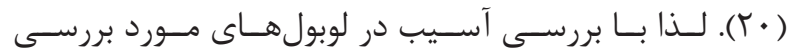

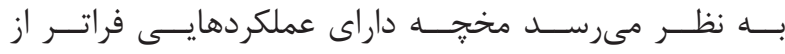
تنظيـم تعـادل، حفـظ تونـوس عضلانـى و هماهنَحَى اعمـال

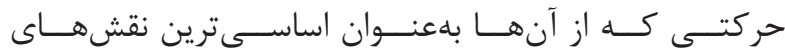

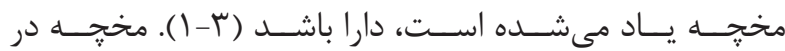

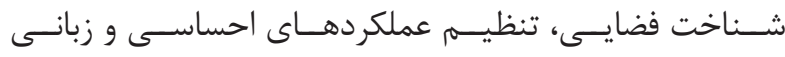

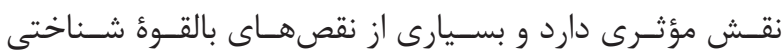

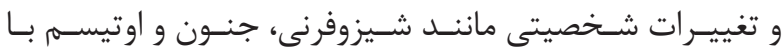

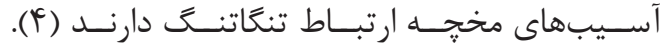

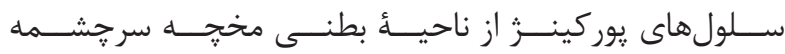

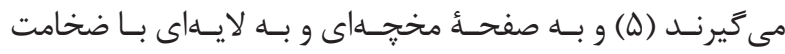

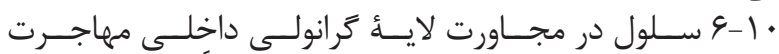

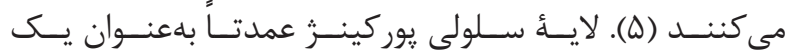

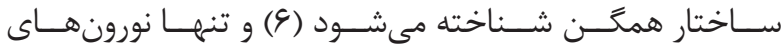

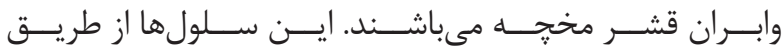

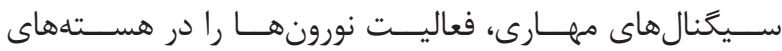

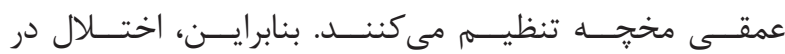

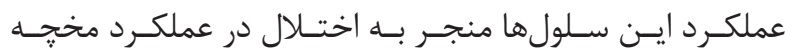

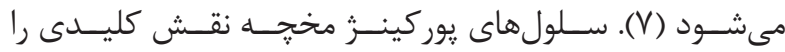

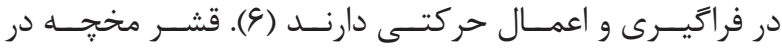

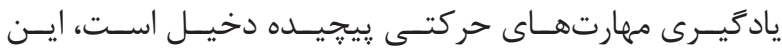

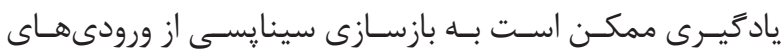

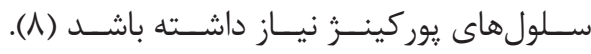

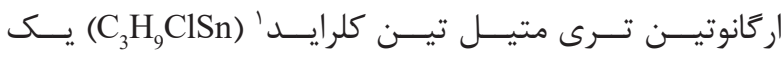

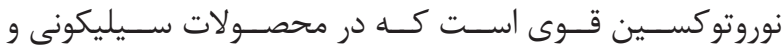

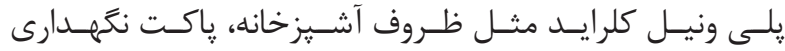

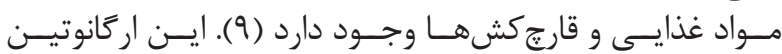

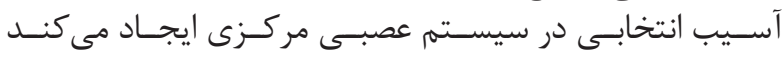

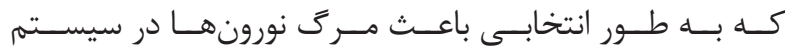

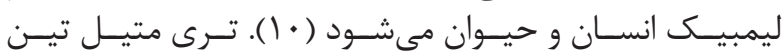

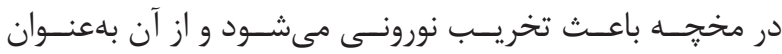

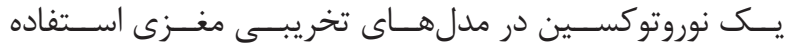

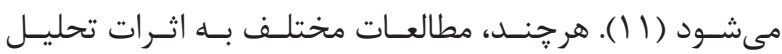

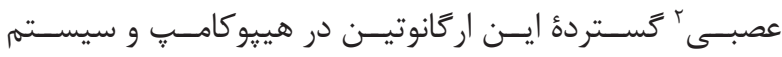

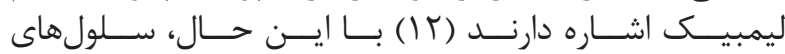

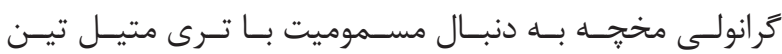

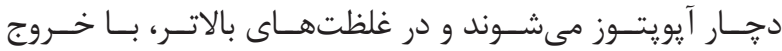

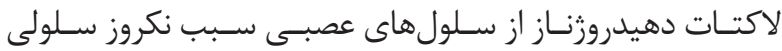

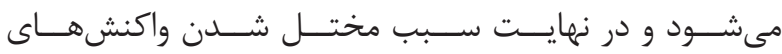

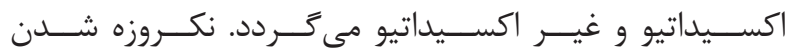

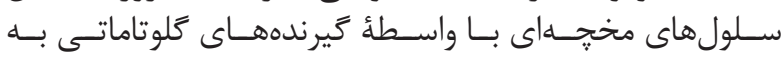

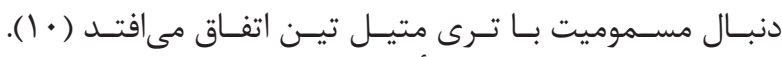

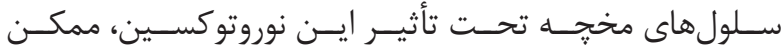

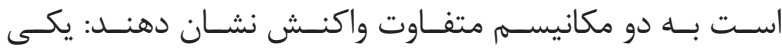

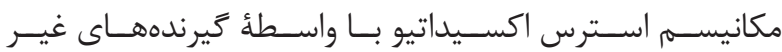

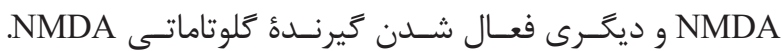




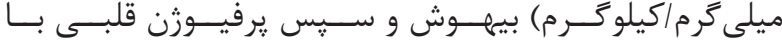

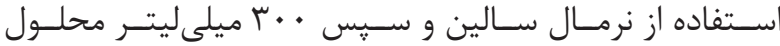

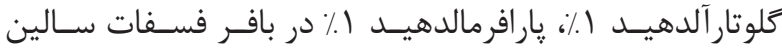

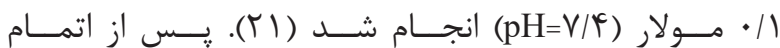

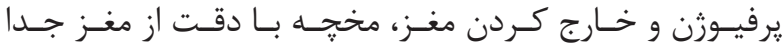

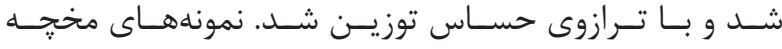

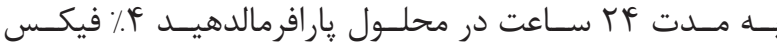

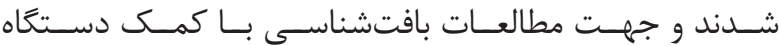

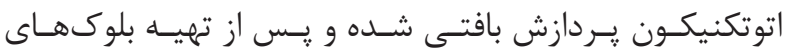

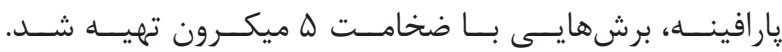

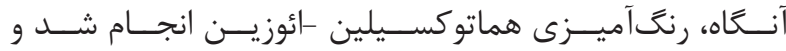

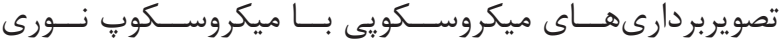
(Olympus-BH $\left.{ }_{2}\right)$ شمارش سلول هاى يور كينز

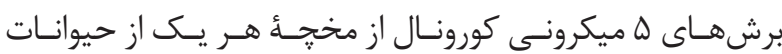

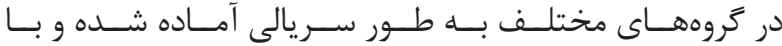

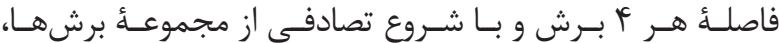

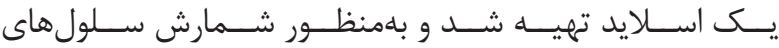

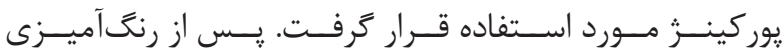

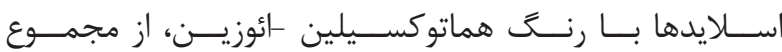

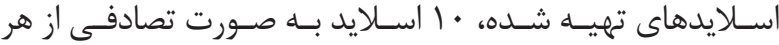

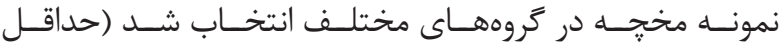

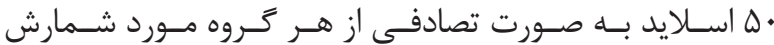

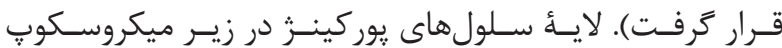

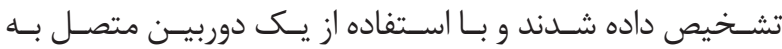

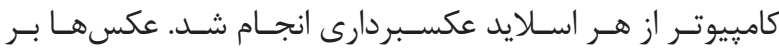

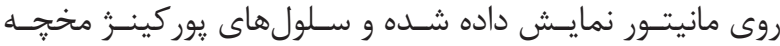

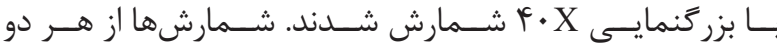

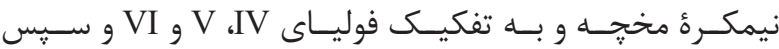

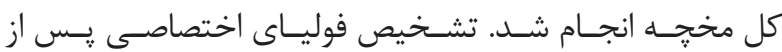

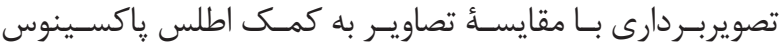

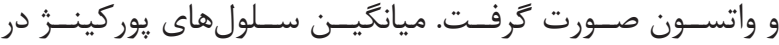

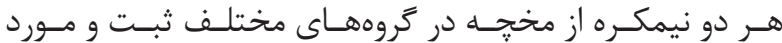

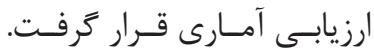

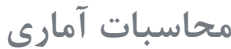

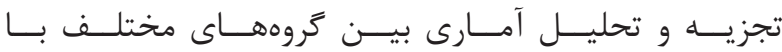

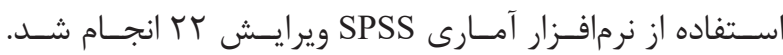

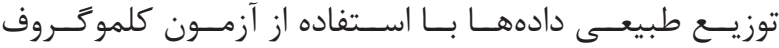

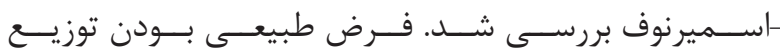

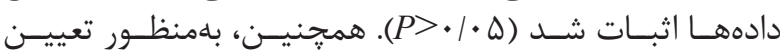

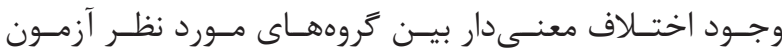

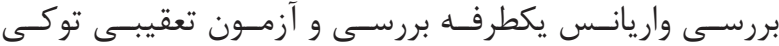

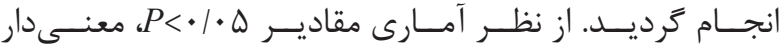

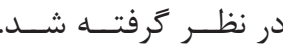

يافتهها

توزين مغز و مخجه و بررسى نسبت بين آنها

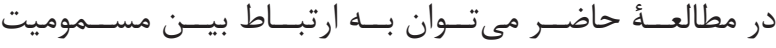

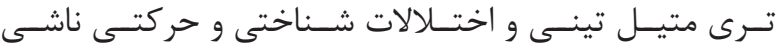

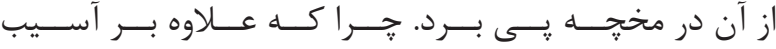

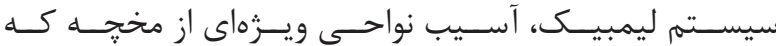

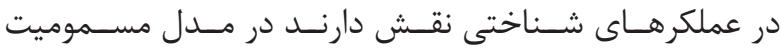

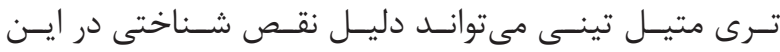

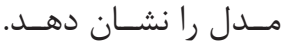

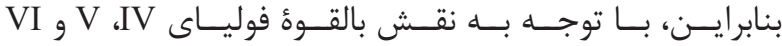

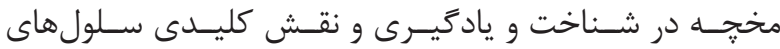

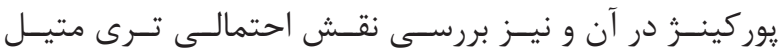

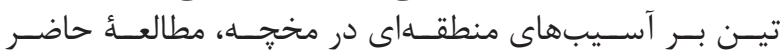

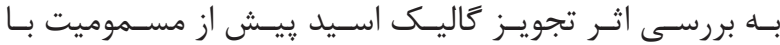

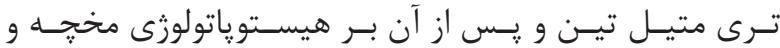

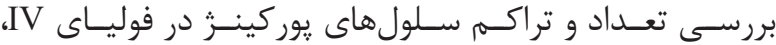

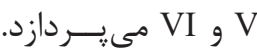

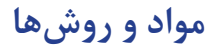

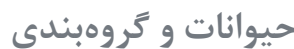

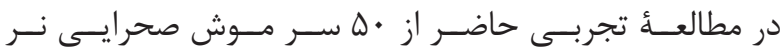

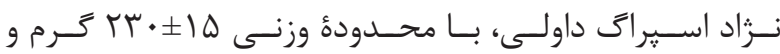

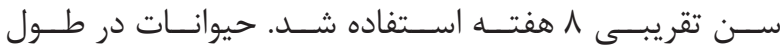

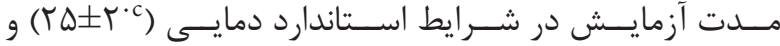

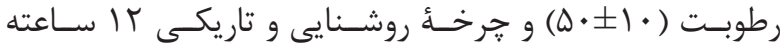

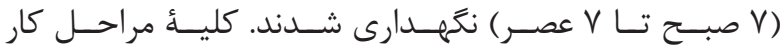

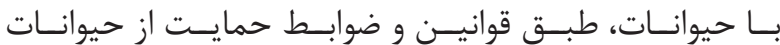

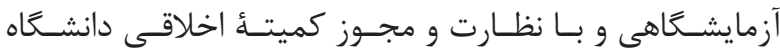

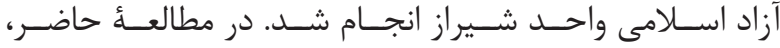

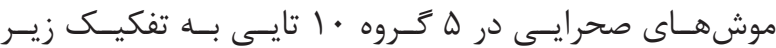

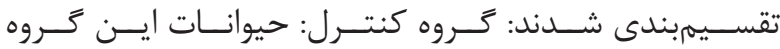

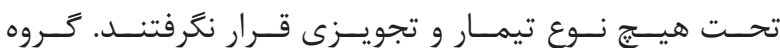

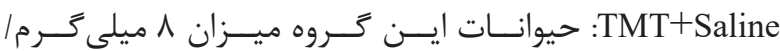

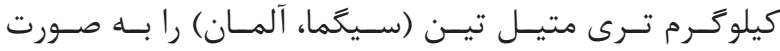

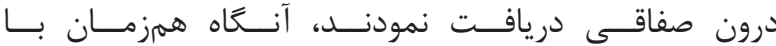

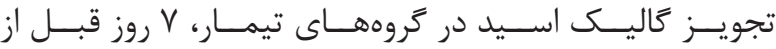

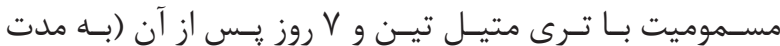

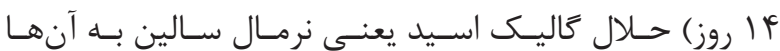

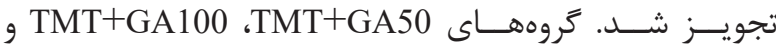

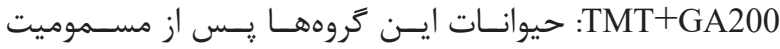

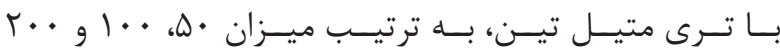

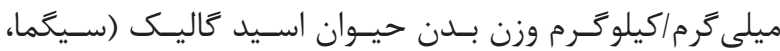

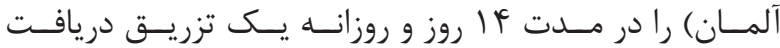

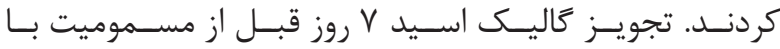

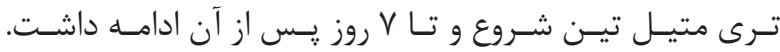

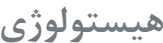

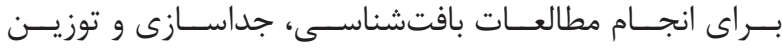

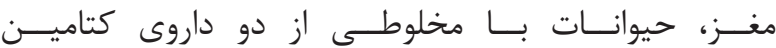

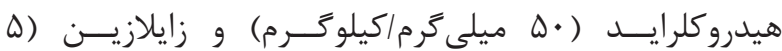


شمارش سلولهاى يور كينز

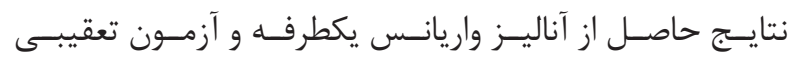

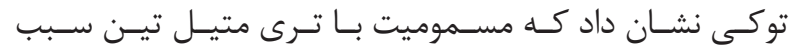

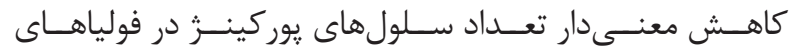

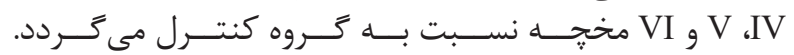

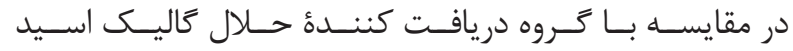

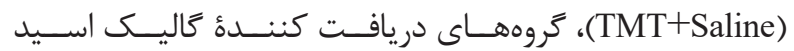

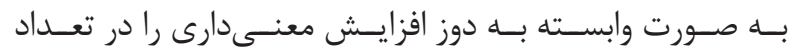

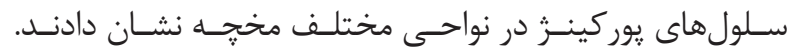

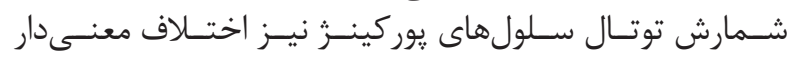

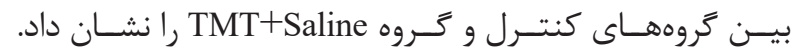

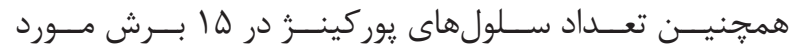

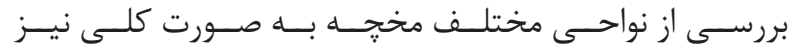

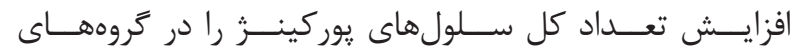

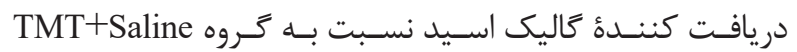

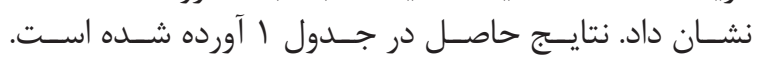

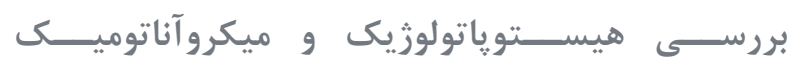

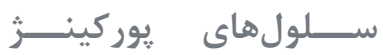

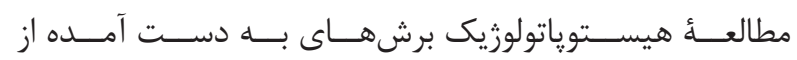

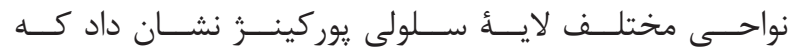

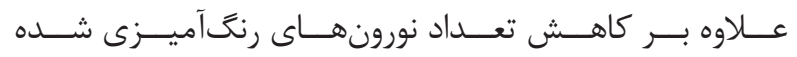

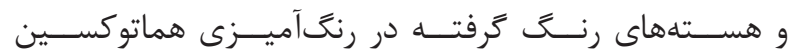

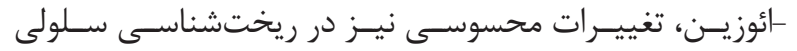

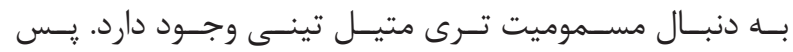

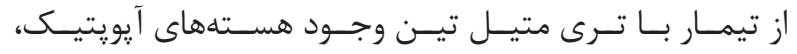

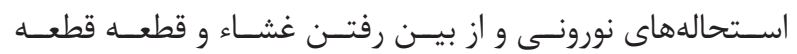

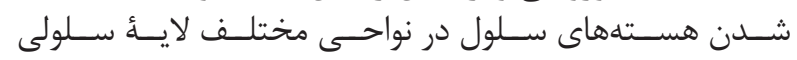

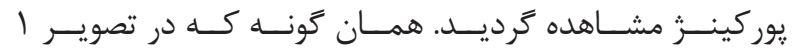

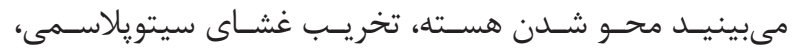

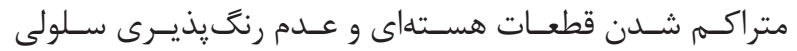

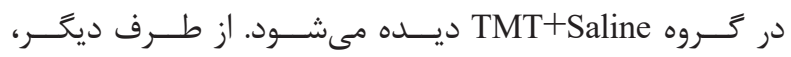

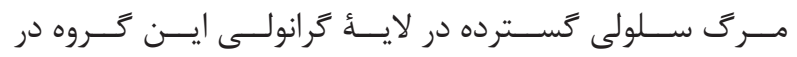

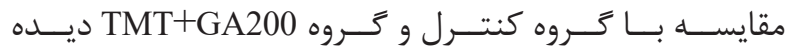

$$
\text { مى شَــــون. }
$$

نتايـج حاصـل از توزيــن مخجهـهـ (نمـودار ا ـالــف) نشــان

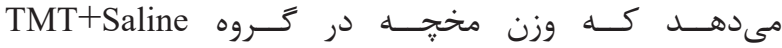
( )

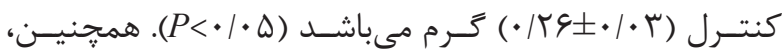

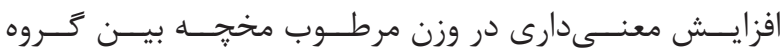
TMT+Saline و كرون

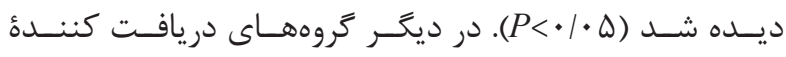

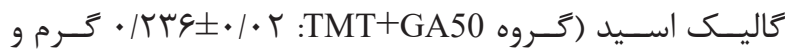

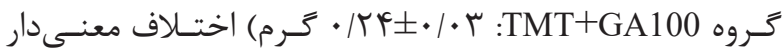

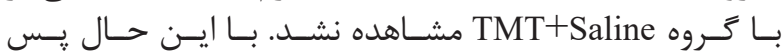

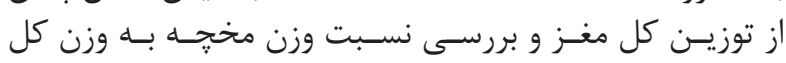

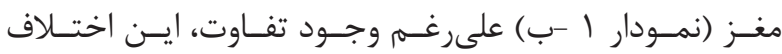

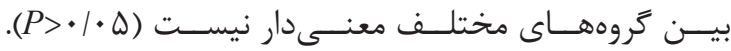
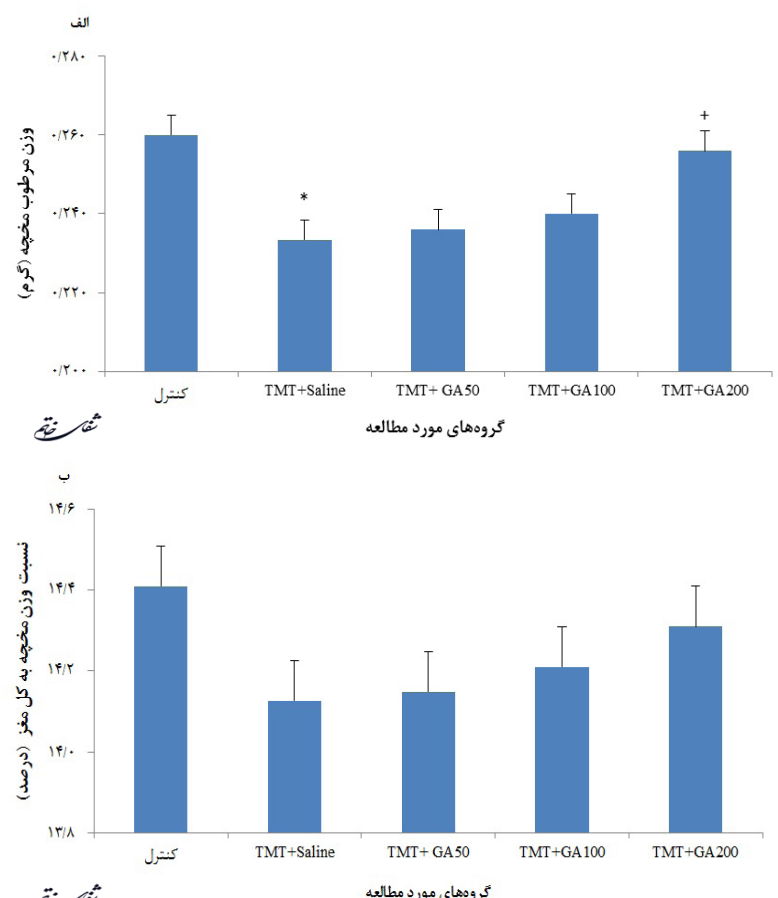
ثم

$$
\text { كروههاي مورن مطالعه }
$$

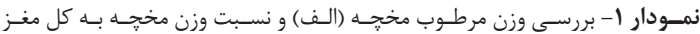

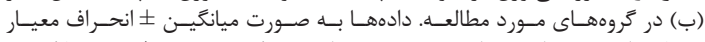

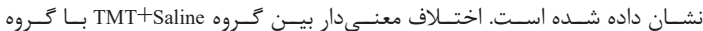

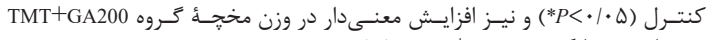

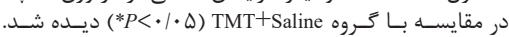

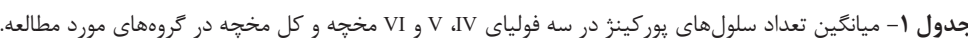

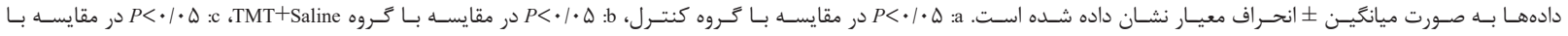

\begin{tabular}{|c|c|c|c|c|}
\hline كل مخجهه & فوليوم VI & فوليوم V & فوليوم IV & تروه \\
\hline$r r \Delta / r \pm r r / \omega$ & $q / V \pm 1 / 4 \Delta$ & $\Lambda / r \pm 1 / \Delta \varphi$ & $N / \boldsymbol{F} \pm 1 / r r$ & كنترل \\
\hline$a<\in / \Lambda \pm q / \Lambda$ & ${ }^{a} \mid / \xi \pm \cdot / r 1$ & ${ }^{\mathrm{a}} / / \cdot 9 \pm \cdot / 1 \mathrm{r}$ & $\mathrm{a} \mathbf{T} / \mathrm{r} \pm \cdot / \mathrm{r}$ & TMT+Saline \\
\hline${ }^{a} \Delta r / r \pm 11 / r$ & a $Y / \Lambda \pm \cdot / M r$ & a $r / \xi \pm \cdot / r$. & $a^{a} / \boldsymbol{F} \pm \cdot / r \wedge$ & $\mathrm{TMT}+\mathrm{GA} 50$ \\
\hline a & a $r / \zeta \pm \cdot / \xi \varphi$ & $\left.{ }^{a b} \boldsymbol{F} / \Lambda \pm \cdot / \bigvee\right)$ & $\mathrm{ab}^{\mathrm{a}} / \wedge \pm \cdot / \Gamma$. & $\mathrm{TMT}+\mathrm{GA} 100$ \\
\hline bcd $Y Y \Delta / \vee \pm Y Y / \Lambda$ & $\operatorname{bcd} \boldsymbol{\varphi} / 9 \pm 1 / 1$ & bcd $V / r \pm 1 / \Delta$ & $\operatorname{bcd} / / \pm 1 / r$ & TMT+GA200 \\
\hline$\cdot 1 \cdots \cdot 1$ & $\cdot 1 \cdot \omega$ & $\cdot 1 \cdots 9$ & $\cdot 1 \cdot \cdots 1$ & سطح معنى دارى \\
\hline
\end{tabular}

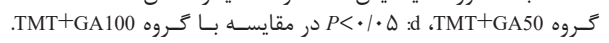



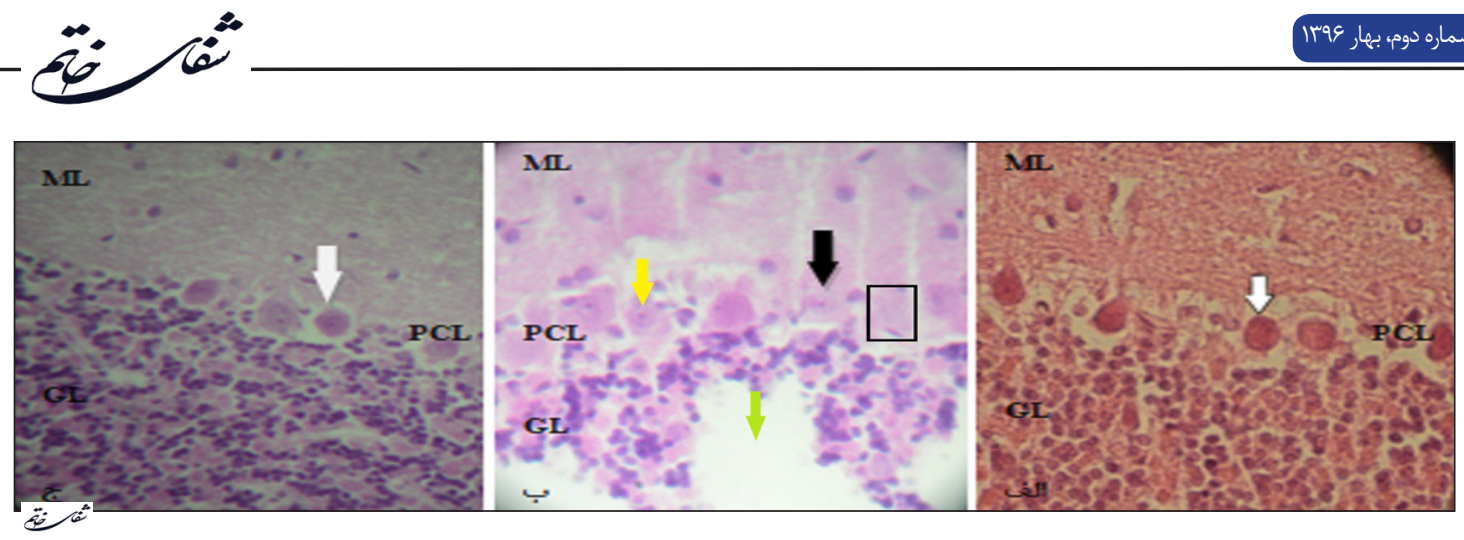

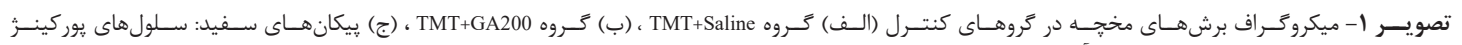

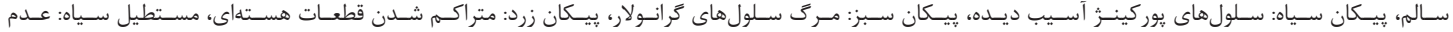

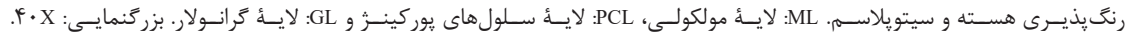

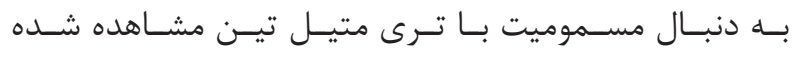

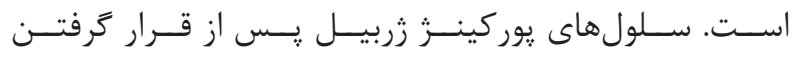

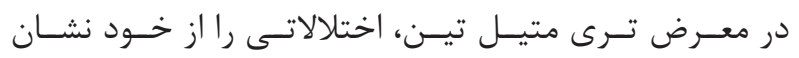

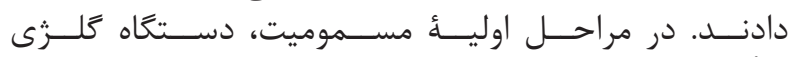

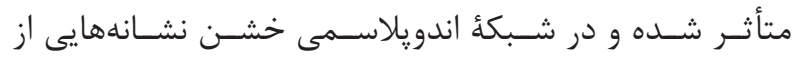

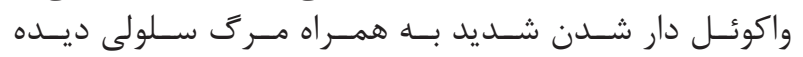

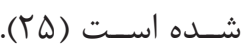

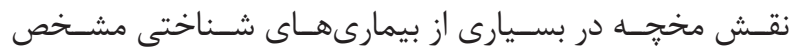

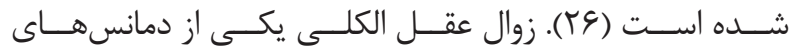

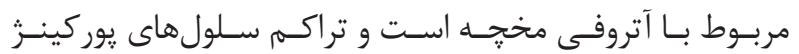

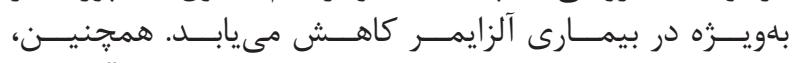

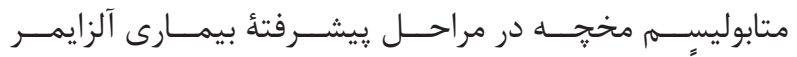

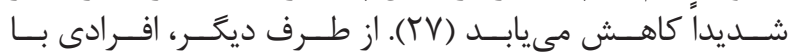

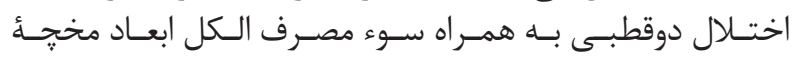

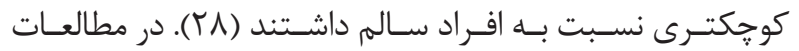

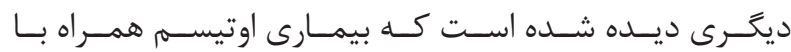

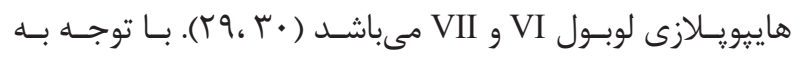

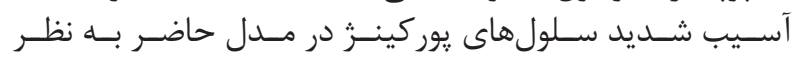

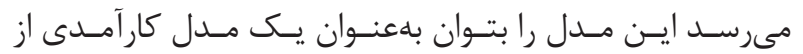

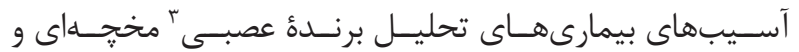

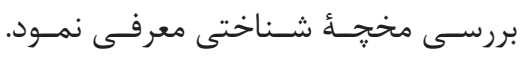

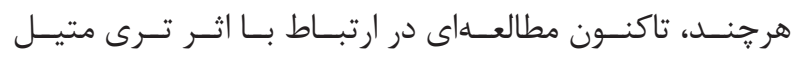

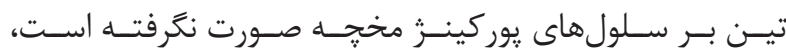

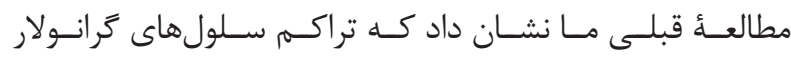

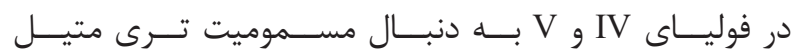

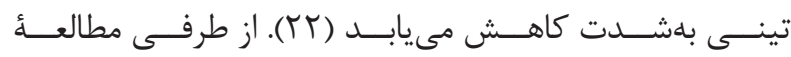

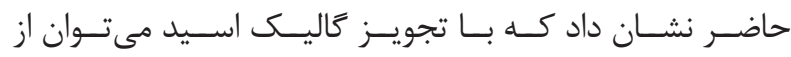

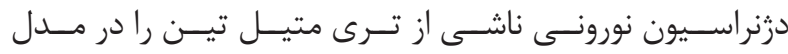

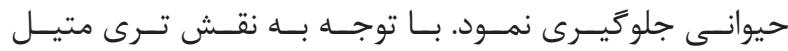

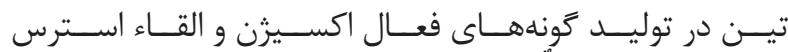

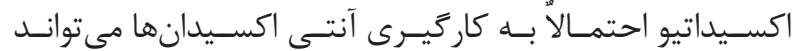

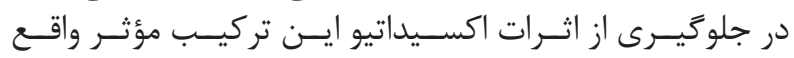

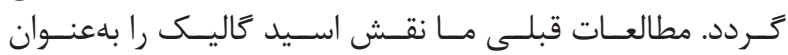

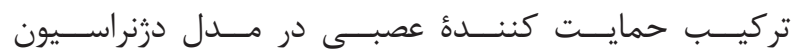

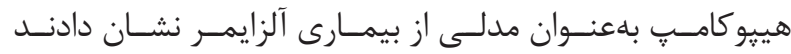

بحث و نتيجه

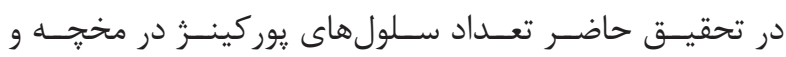

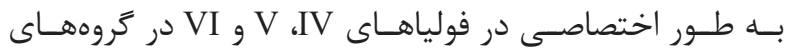

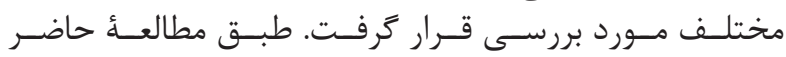

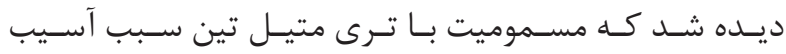

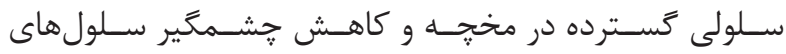

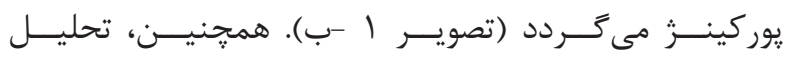

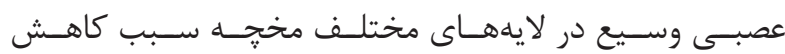

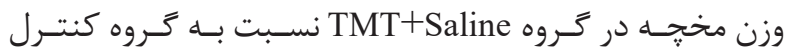

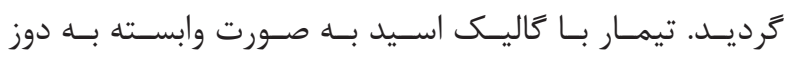

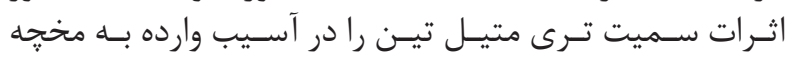

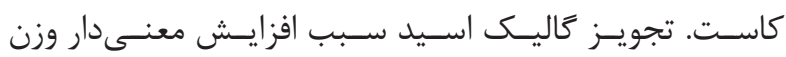

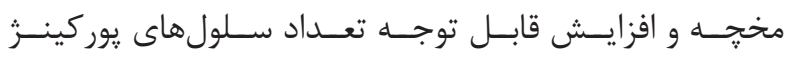

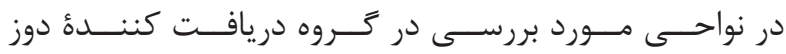

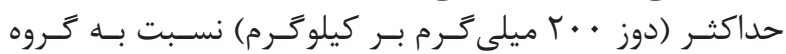
كرديـد TMT+saline

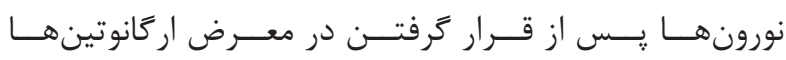

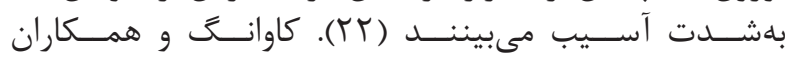

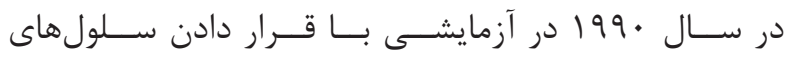

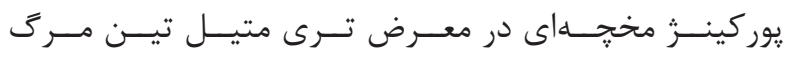

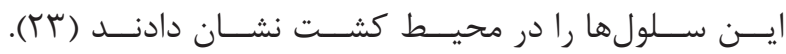

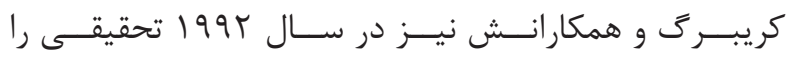

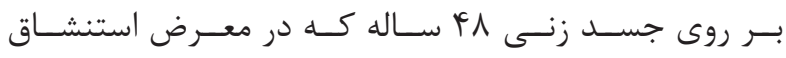

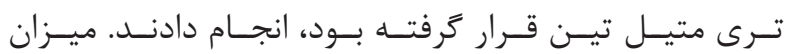

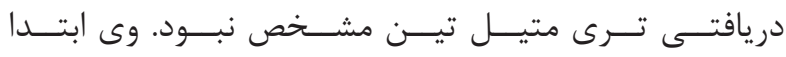

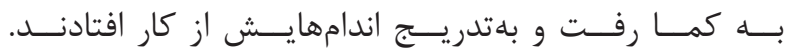

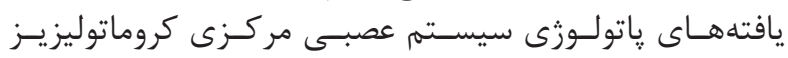

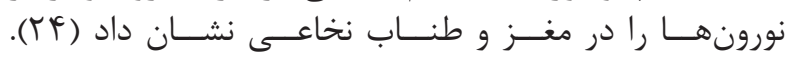

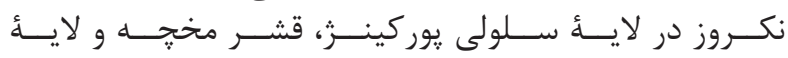

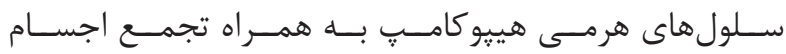

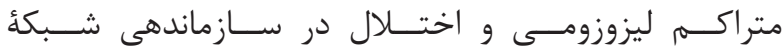

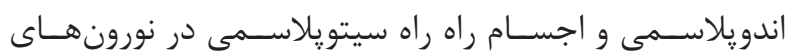

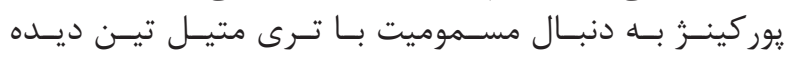

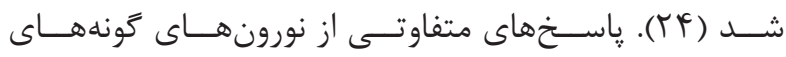

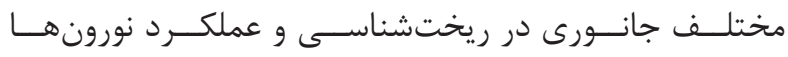




$$
\begin{aligned}
& \text { را در كاهـش اثـرات ســميت سـلولى و مــرك نورونسى تـرى }
\end{aligned}
$$

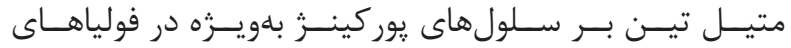

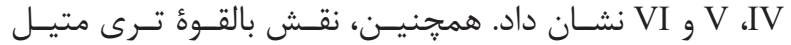

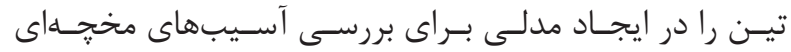

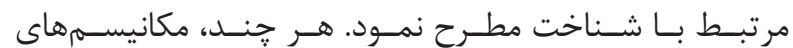

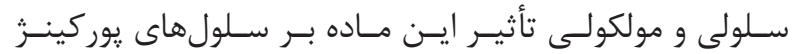

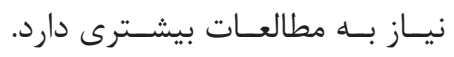

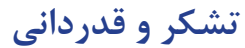

1. Albus JS. A theory of cerebellar function. Math Biosci. 1971; 10(1-2): 25-61.

2. Armstrong DM, Harvey RJ, Schild RF. Topographical localization in the olivo-cerebellar projection: an electrophysiological study in the cat. J Comp Neurol. 1974; 154(3): 287-302.

3. Cesa R, Strata P. Axonal competition in the synaptic wiring of the cerebellar cortex during development and in the mature cerebellum. Neuroscience. 2009; 162(3): 624-32.

4. Hoche F, Guell X, Sherman JC, Vangel MG, Schmahmann JD. Cerebellar contribution to social cognition. Cerebellum. 2016; 15(6): 732-43.

5. Medini C, Nair B, D’Angelo E, Naldi G, Diwakar $\mathrm{S}$. Modeling spike-train processing in the cerebellum granular layer and changes in plasticity reveal single neuron effects in neural ensembles. Computat Intelli Neurosci. 2012; 35(9): 5. Doi: 10.1155/2012/359529.

6. Porras-Garcia ME, Ruiz R, Perez-Villegas EM, Armengol JA. Motor learning of mice lacking cerebellar purkinje cells. Front Neuroanat. 2013; 90(5): 7-13.

7. Bernard JA, Seidler RD, Hassevoort KM, Benson BL, Welsh RC, Wiggins JL, et al. Resting state cortico-cerebellar functional connectivity networks: a comparison of anatomical and self-organizing map approaches. Front Neuroanat. 2012; 6(4): 120-8.

8. Sullivan EV. Cognitive functions of the cerebellum. Neuropsychol Rev. 2010; 20(3): 227-8.

9. Geloso MC, Corvino V, Michetti F. Trimethyltin-

${ }^{4}$ Glutathione peroxidase

${ }^{5}$ Malondialdehyde

${ }^{6}$ Neuroprotection

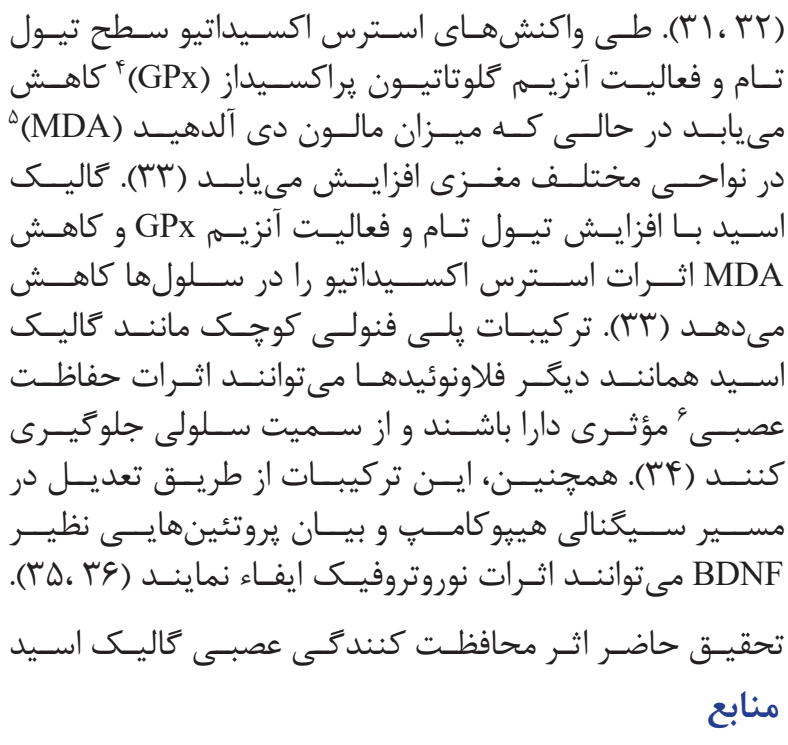

induced hippocampal degeneration as a tool to investigate neurodegenerative processes. Neurochemistry International. 2011; 58(7): 729-38.

10. Gunasekar P, Li L, Prabhakaran K, Eybl V, Borowitz $\mathrm{JL}$, Isom GE. Mechanisms of the apoptotic and necrotic actions of trimethyltin in cerebellar granule cells. Toxicological Sciences. 2001; 64(1): 83-9.

11. Haga S, Haga C, Aizawa T, Ikeda K. Neuronal degeneration and glial cell-responses following trimethyltin intoxication in the rat. Acta Neuropathol. 2002; 103(6): 575-82.

12. Gasparova Z, Pronayova N, Stara V, Liptaj T. In vitro metabolomic approach to hippocampal neurodegeneration induced by trimethyltin. Neurochem Res. 2016; 41(4): 715-21.

13. Reuhl KR, Gilbert SG, Mackenzie BA, Mallett JE, Rice DC. Acute trimethyltin intoxication in the monkey (Macaca fascicularis). Toxicol Appl Pharmacol. 1985; 79(3): 436-52.

14. Anderson VE, Hajimohammadreza I, Gallo JM, Anderton BH, Uney J, Brown AW, et al. Ubiquitin, PGP 9.5 and dense body formation in trimethyltin intoxication: differential neuronal responses to chemically induced cell damage. Neuropathol Appl Neurobiol. 1992; 18(4): 360-75.

15. Alasalvar C, Bolling BW. Review of nut phytochemicals, fat-soluble bioactives, antioxidant components and health effects. Br J Nutr. 2015; 113(2): S68-78.

16. Choubey S, Varughese LR, Kumar V, Beniwal $\mathrm{V}$. Medicinal importance of gallic acid and its ester 


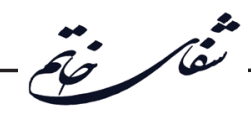

derivatives: a patent review. Pharm Pat Anal. 2015; 4(4): 305-15.

17. Sarjit A, Wang Y, Dykes GA. Antimicrobial activity of gallic acid against thermophilic Campylobacter is strain specific and associated with a loss of calcium ions. Food Microbiol. 2015; 46: 227-33.

18. Hsieh SC, Wu CC, Hsu SL, Yen JH. Molecular mechanisms of gallic acid-induced growth inhibition, apoptosis, and necrosis in hypertrophic scar fibroblasts. Life Sci. 2016; pii: S0024-3205(16)30468-4.

19. Stoodley CJ, Schmahmann JD. Functional topography in the human cerebellum: a meta-analysis of neuroimaging studies. NeuroImage. 2009; 44(2): 489-501.

20. Schmahmann JD. Disorders of the cerebellum: ataxia, dysmetria of thought, and the cerebellar cognitive affective syndrome. J Neuropsychiatry Clin Neurosci. 2004; 16: 367-78.

21. Edalatmanesh MA, Bahrami AR, Hosseini E, Hosseini M, Khatamsaz S. Bone marrow derived mesenchymal stem cell transplantation in cerebellar degeneration: a behavioral study. Behav Brain Res. 2011; 225(1): 63-70.

22. Shams-Alam S, Edalatmanesh MA. The effete of lithium chloride on the granular cell density in cerebellar folia V and VI in a trimethyltin intoxication model. Shefaye Khatam. 2015; 3(2): 41-8.

23. Cavanagh JB, Nolan CC, Brown AW. Glial cell intrusions actively remove detritus due to toxic chemicals from within nerve cells. Neurotoxicology. 1990; 11(1): 1-12.

24. Kreyberg S, Torvik A, Bjørneboe A, Wiik-Larsen W, Jacobsen D. Trimethyltin poisoning: report of a case with postmortem examination. Clin Neuropathol. 1991; 11(5): 256-9.

25. Nolan CC, Brown AW, Cavanagh JB. Regional variations in nerve cell responses to trimethyltin intoxication in mongolian gerbils and rats; further evidence for involvement of the golgi apparatus. Acta Neuropathol. 1990; 81(2): 204-12.

26. Bodranghien F, Bastian A, Casali C, Hallett M, Louis ED, Manto M, et al. consensus paper: revisiting the symptoms and signs of cerebellar syndrome.
Cerebellum. 2016; 15(3): 369-91.

27. Schmahmann JD. Cerebellum in Alzheimer's disease and frontotemporal dementia: not a silent bystander. Brain. 2016; 139(5): 1314-8.

28. Zhao L, Wang Y, Jia Y, Zhong S, Sun Y, Zhou Z, et al. Cerebellar microstructural abnormalities in bipolar depression and unipolar depression: a diffusion kurtosis and perfusion imaging study. J Affect Disord. 2016; 195: 21-31.

29. Jaber M. The cerebellum as a major player in motor disturbances related to autistic syndrome disorders. encephale. 2016; pii: S0013-7006(16)30132-4.

30. Rout UK, Dhossche DM. A pathogenetic model of autism involving Purkinje cell loss through anti-GAD antibodies. Medical Hypotheses. 2008; 71: 218-21.

31. Bazyar Y, Rafiei S, Hosseini A, Edalatmanesh MA. Effect of endurance exercise training and gallic acid on tumor necrosis factor- $\alpha$ in an animal model of Alzheimer's disease. Shefaye Khatam. 2015; 3(3): 21-6.

32. Rafiei S, Bazyar Y, Edalatmanesh MA. Effect of gallic acid and endurance exercise training on BDNF in a model of hippocampal degeneration. Shefaye Khatam. 2016; 4(1):1-6.

33. Mansouri MT, Farbood Y, Sameri MJ, Sarkaki A, Naghizadeh B, Rafeirad M. Neuroprotective effects of oral gallic acid against oxidative stress induced by 6-hydroxydopamine in rats. Food Chem. 2013; 138(23): $1028-33$.

34. Daglia M, Di Lorenzo A, Nabavi SF, Talas ZS, Nabavi SM. Polyphenols: well beyond the antioxidant capacity: gallic acid and related compounds as neuroprotective agents: you are what you eat! Curr Pharm Biotechnol. 2014; 15(4): 362-72.

35. Corona G, Vauzour D, Hercelin J, Williams CM, Spencer JP. Phenolic acid intake, delivered via moderate champagne wine consumption, improves spatial working memory via the modulation of hippocampal and cortical protein expression/activation. Antioxid Redox Signal. 2013; 19(14): 1676-89.

36. Moosavi F, Hosseini R, Saso L, Firuzi O. Modulation of neurotrophic signaling pathways by polyphenols. Drug Des Devel Ther. 2015; 10: 23-42. 